\title{
Rehmet, Frank, Neelke Wagner, und Tim Willy Weber (2019): Volksabstimmungen in Europa. Regelungen und Praxis im internationalen Vergleich
}

\author{
Opladen: Verlag Barbara Budrich. 202 Seiten. $26 €$
}

\section{Arndt Leininger}

Online publiziert: 15 . Juli 2020

(C) Der/die Autor(en) 2020

Mit der gestiegenen Zahl an Verfahren direkter Demokratie in den letzten Jahren und Jahrzehnten nahm auch das politikwissenschaftliche Interesse an diesen stetig zu. Jenseits von Studien zu einzelnen Abstimmungen steht die komparative Forschung vor der Herausforderung, die große Vielfalt an Verfahren zu erfassen, die zudem von Land zu Land, aber auch von Autor zu Autorin in den Gesellschaftswissenschaften unterschiedlich benannt werden.

Hier verspricht ein neues Überblickswerk Orientierung zu geben. Die AutorInnen Frank Rehmet, Neelke Wagner und Tim Willy Weber sind aktuelle und ehemalige MitarbeiterInnen des Vereins „Mehr Demokratie e.V.“, der die jedes zweite Jahr im Wechsel erscheinenden Volks- und Bürgerbegehrensberichte herausgibt. Diese gehören zu den wichtigsten Informationsquellen zum aktuellen Stand der direkten Demokratie in den deutschen Ländern und Kommunen. Mit „Volksabstimmungen in Europa. Regelungen und Praxis im internationalen Vergleich" haben die AutorInnen nun einen europäischen Überblick zu nationalen Volksabstimmungen, mit ähnlichem Potenzial zu einem einschlägigen Referenzwerk zu werden, vorgelegt.

Die Unterüberschrift „Regelungen und Praxis“ ist passend gewählt, da sich direktdemokratische Verfahren in Regelungen und Praxis grundlegend unterscheiden können. So gibt es auch Länder wie zum Beispiel Großbritannien mit Praxis aber ohne feststehende verfassungsrechtliche oder gesetzliche Regelungen. Das von den AutorInnen beschriebene Europa umfasst neben den 27 Mitgliedsstaaten der Europäischen Union auch die Länder Albanien, Andorra, Bosnien und Herzegowina, Großbritannien, Island, Liechtenstein, Kosovo, Moldawien, Monaco, Montenegro, Nordmazedonien, Norwegen, San Marino, die Schweiz, Serbien und die Ukraine. Die AutorInnen berücksichtigen alle in diesen Ländern seit Gründung als demo-

\footnotetext{
A. Leininger $(\bowtie)$

Otto-Suhr-Institut für Politikwissenschaft, Freie Universität Berlin, Berlin, Deutschland

E-Mail: arndt.leininger@fu-berlin.de
} 
kratische Staaten stattgefundenen Volksabstimmungen. Für die Schweiz beginnt der Beobachtungszeitraum also schon 1848, für Deutschland 1949 und für die Staaten Osteuropas 1990.

Das Buch soll eine ,wachsende Nachfrage nach Informationen über Volksabstimmungen“ (S. 7) bedienen: Fragen wie „Wie viele Länder in Europa kennen eigentlich Volksabstimmungen? Wo sind welche Verfahren vorhanden und wie oft werden sie genutzt?" (S. 7) sollen durch das Buch beantwortet werden. Neben der Deskription bestehender Regelungen und vergangener Abstimmungen, welche den weit überwiegenden Teil des Buches einnimmt, besteht ein weiteres Ziel der VerfasserInnen darin, die bestehenden Regelungen und die darauf aufbauende Praxis in den betrachteten Ländern zu evaluieren.

Die große Stärke des Buches liegt eindeutig in ersterem, der begrifflichen und empirischen Aufbereitung des Themenfeldes direkter Demokratie. Im ersten Kapitel widmen sich Rehmet, Wagner und Weber einer Definition und Typologisierung der betrachteten Verfahren, welche sich an einschlägigen Beiträgen aus der Politikwissenschaft orientiert und durch eine Vielzahl an Tabellen und Abbildungen anschaulich dargestellt wird. Im Einklang mit dem Konsens in der politikwissenschaftlichen Fachliteratur fokussieren die AutorInnen auf Abstimmungen durch BürgerInnen über politische Sachfragen. Direktwahlen oder die Abwahl von AmtsträgerInnen, aber auch Petitionsverfahren, die lediglich die Befassung von Parlamenten mit einem Thema, aber keine Volksabstimmung nach sich ziehen, werden nicht berücksichtigt.

Die VerfasserInnen definieren den Begriff ,direkte Demokratie“ sehr eng. Sie verstehen darunter ausschließlich Verfahren, die durch BürgerInnen initiiert werden oder durch gesetzliche Automatismen ausgelöst werden und deren Abstimmungsergebnis verbindlich ist. Diese Definition wird sicherlich nicht bei allen LeserInnen auf Zustimmungen stoßen, deckt sie sich doch nicht mit den mitunter breiteren Begriffsverständnissen in der Fachliteratur. Jedoch berücksichtigen die AutorInnen auch durch Parlamente und Regierung ausgelöste Verfahren sowie unverbindliche Abstimmungen. Angesichts der klaren Begriffsdefinitionen im ersten Kapitel werden LeserInnen keine Schwierigkeiten haben, die sie interessierenden Verfahren im Buch aufzufinden.

Das zweite Kapitel gibt dann einen knappen Gesamtüberblick über bestehende Regelungen und vergangene Abstimmungen in Europa. Den größten Teil des Buches nimmt das dritte Kapitel ein, in dem nach Nutzungshäufigkeit sortierte Ländergruppen in Unterkapiteln vorgestellt werden. Innerhalb dieser Unterkapitel werden für jedes Land die bestehenden Regelungen und bisherige Verfahren dargestellt und kommentiert. Zudem wird, soweit vorhanden, auf weitere Datenquellen und teilweise auch weiterführende Literatur verwiesen. Hier liegt die größte Stärke des Buches als Nachschlagewerk.

Weniger zu überzeugen vermag der evaluative Aspekt des Buches. Schon die von Rehmet, Wagner und Weber aufgestellten „Qualitätsmerkmale“ (S. 19-20), anhand derer die VerfasserInnen die beschriebenen Verfahren bewerten wollen, dürften bei vielen LeserInnen Kritik auf sich ziehen. Das Vorhandensein klarer und gesetzlich normierter Regeln kann noch als allgemein wünschenswert und unumstritten gelten. Anders sieht es aus mit Forderungen, wie zum Beispiel, dass es keine Themenausschlüsse geben dürfe oder Volksabstimmungen Parlamentsbeschlüssen gleichgestellt 
sein müssten. Dies wird sicherlich Widerspruch provozieren. Zumal diese Kriterien teils gar nicht oder nur sehr knapp und ohne Rückbezug auf Forschungsliteratur, die es durchaus gibt, erläutert werden. Andere Anforderungen wie „Unterschriften[quoren, die] den Bürger/innen eine realistische Chance einräumen, eine Volkabstimmung auch auslösen zu können“ (S. 20) bleiben schlicht zu vage.

So wirken auch die im letzten Kapitel anhand der eingangs aufgestellten Kriterien identifizierten Best und Worst Practices stark subjektiv. Man merkt dem Buch auch hier an, dass die AutorInnen VerfechterInnen direktdemokratischer Verfahren sind. Man muss jedoch die durchaus kritikwürdigen evaluativen und normativen Ansichten von Rehmet, Wagner und Weber nicht teilen, um dieses akribisch recherchierte Buch mit Gewinn zu lesen. Das umfangreiche Datenmaterial zu über 1000 Abstimmungen, aufbereitet in 65 Tabellen und 18 Abbildungen, macht das Buch zu einem exzellenten deutschsprachigen Nachschlagewerk für an direkter Demokratie interessierte PolitikwissenschaftlerInnen.

Funding Open Access funding provided by Projekt DEAL.

Open Access Dieser Artikel wird unter der Creative Commons Namensnennung 4.0 International Lizenz veröffentlicht, welche die Nutzung, Vervielfältigung, Bearbeitung, Verbreitung und Wiedergabe in jeglichem Medium und Format erlaubt, sofern Sie den/die ursprünglichen Autor(en) und die Quelle ordnungsgemäß nennen, einen Link zur Creative Commons Lizenz beifügen und angeben, ob Änderungen vorgenommen wurden.

Die in diesem Artikel enthaltenen Bilder und sonstiges Drittmaterial unterliegen ebenfalls der genannten Creative Commons Lizenz, sofern sich aus der Abbildungslegende nichts anderes ergibt. Sofern das betreffende Material nicht unter der genannten Creative Commons Lizenz steht und die betreffende Handlung nicht nach gesetzlichen Vorschriften erlaubt ist, ist für die oben aufgeführten Weiterverwendungen des Materials die Einwilligung des jeweiligen Rechteinhabers einzuholen.

Weitere Details zur Lizenz entnehmen Sie bitte der Lizenzinformation auf http://creativecommons.org/ licenses/by/4.0/deed.de. 